\title{
Non-muscle myosin II drives vesicle loss during human reticulocyte maturation
}

\section{Pedro L. Moura, ${ }^{1}$ Bethan R. Hawley, ${ }^{1}$ Tosti J. Mankelow, ${ }^{2,3}$ \\ Rebecca E. Griffiths, ${ }^{2,3,4}$ Johannes G.G. Dobbe ${ }^{5}$ Geert J. Streekstra, ${ }^{5}$ David J. Anstee,,$^{2,3}$ Timothy J. Satchwell ${ }^{1,2,3^{*}}$ and Ashley M. Toye $\mathrm{e}^{1,2,3^{*}}$}

${ }^{1}$ School of Biochemistry, University of Bristol, UK; ${ }^{2}$ Bristol Institute for Transfusion Sciences, National Health Service Blood and Transplant (NHSBT), UK; ${ }^{3} \mathrm{NIHR}$ Blood and Transplant Research Unit, University of Bristol, UK; ${ }^{4}$ UQ-StemCARE, Australian Institute for Bioengineering and Nanotechnology, The University of Queensland, Australia and ${ }^{5}$ Department of Biomedical Engineering and Physics, Academic Medical Center, University of Amsterdam, the Netherlands

*These authors contributed equally to this work

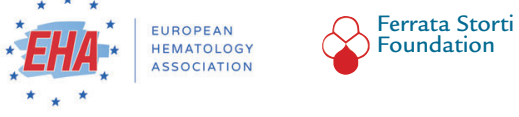

Haematologica 2018

Volume 103(12):1997-2007

\section{ABSTRACT}

T The process of maturation of reticulocytes into fully mature erythrocytes that occurs in the circulation is known to be characterized by a complex interplay between loss of cell surface area and volume, removal of remnant cell organelles and redundant proteins, and highly selective membrane and cytoskeletal remodeling. However, the mechanisms that underlie and drive these maturational processes in vivo are currently poorly understood and, at present, reticulocytes derived through in vitro culture fail to undergo the final transition to erythrocytes. Here, we used high-throughput proteomic methods to highlight differences between erythrocytes, cultured reticulocytes and endogenous reticulocytes. We identify a cytoskeletal protein, non-muscle myosin IIA (NMIIA) whose abundance and phosphorylation status differs between reticulocytes and erythrocytes and localized it in the proximity of autophagosomal vesicles. An ex vivo circulation system was developed to simulate the mechanical shear component of circulation and demonstrated that mechanical stimulus is necessary, but insufficient for reticulocyte maturation. Using this system in concurrence with non-muscle myosin II inhibition, we demonstrate the involvement of non-muscle myosin IIA in reticulocyte remodeling and propose a previously undescribed mechanism of shear stress-responsive vesicle clearance that is crucial for reticulocyte maturation.

\section{Introduction}

Reticulocytes are anucleate erythroid cells which undergo maturation to form the biconcave erythrocyte. ${ }^{1}$ The mechanism leading to maturation is relatively undefined, but it is known to occur in the peripheral circulation within 1-2 days after cells have egressed from the bone marrow. ${ }^{2}$ As the current endpoint of existing in vitro erythroid culture systems, ${ }^{3,4}$ interest in this cell type and the mechanisms and factors that may underlie and drive their maturation to erythrocytes has received renewed interest in recent years.

The phenotypic differences between reticulocytes and erythrocytes have been studied in detail. Reticulocyte maturation involves extensive membrane and cytoskeletal remodeling, with loss of approximately $20 \%$ of cell surface area during this process ${ }^{5.9}$ which allows the initially amorphous reticulocyte to acquire the characteristic erythrocyte biconcave morphology and accompanying increased resistance to shear stress. This remodeling is a highly selective process, characterized by proteasomal degradation and exocytosis of specific components (e.g. actin, myosin, talin) with preferential retention of the remainder (e.g. $\alpha$ or $\beta$-spectrin). ${ }^{7}$ Other notable hallmarks of maturation include a progressive loss of RNA content, an increase in cell deformability, and a decrease in both cytoplasmic and surface protein content through exocytosis, membrane shedding and autophagy-mediated pathways. ${ }^{601012}$

The most well-described pathway relating to loss of protein during reticulocyte

\section{Correspondence: \\ t.satchwell@bristol.ac.uk or ash.m.toye@bristol.ac.uk}

Received: June 1, 2018.

Accepted: July 26, 2018.

Pre-published: August 3, 2018.

doi:10.3324/haematol.2018.199083

Check the online version for the most updated information on this article, online supplements, and information on authorship \& disclosures: www.haematologica.org/content/103/12/1997

\section{(C)2018 Ferrata Storti Foundation}

Material published in Haematologica is covered by copyright. All rights are reserved to the Ferrata Storti Foundation. Use of published material is allowed under the following terms and conditions:

https://creativecommons.org/licenses/by-nc/4.0/legalcode. Copies of published material are allowed for personal or internal use. Sharing published material for non-commercial purposes is subject to the following conditions:

https://creativecommons. org//icenses/by-nc/4.0/legalcode, sect. 3. Reproducing and sharing published material for commercial purposes is not allowed without permission in writing from the publisher. 
maturation concerns the removal of the transferrin receptor CD71. ${ }^{10}$ This mechanism of plasma membrane protein loss, which involves the internalization of receptors within endocytic vesicles, multivesicular body formation and the release of exosomes has been extensively studied and shown to also mediate the loss of other proteins. ${ }^{12}$ Other major mechanisms involved in the removal of proteins during reticulocyte maturation are encompassed by the process of autophagy. Autophagy occurs through the sorting of redundant, damaged or leftover organelles and other cytoplasmic content into autophagosomes ${ }^{13}$ which in reticulocytes have been reported to fuse with lysosomes and form autophagolysosomes that are later expelled from the cell. ${ }^{4,14}$ Parts of this process are highly specific: for instance, $\mathrm{NIX}^{-}$mice undergo autophagy and maturation, but not mitophagy, causing retention of mitochondria in reticulocytes. ${ }^{15}$

Using reticulocytes cultured in vitro, the autophagosome was recently shown to be involved in the process of remodeling of the reticulocyte membrane which involves the release of an inside-out vesicle. ${ }^{4}$ It is also known that, in other mammalian cells, mechanical stress upregulates autophagy, and the removal of autophagic vesicles in the reticulocyte is triggered by passage through the sinusoidal walls of the spleen. ${ }^{10,17}$ However, the process of autophagic vesicle transport and the eventual release of these vesicles from reticulocytes is poorly understood.

In vitro-derived reticulocytes expanded and differentiated under conditions compatible with clinical use do not currently emulate the final stages of maturation, which occur in vivo after egress from the bone marrow and within the circulation to generate definitive erythrocytes. However, transfusion of in vitro-derived reticulocytes into mouse models induced these final stages of maturation, ${ }^{3,18}$ indicating the involvement of as of yet undefined factors or stimuli not recapitulated in the in vitro culture process. During their time in the peripheral circulation, reticulocytes are exposed to a variety of new stimuli, including shear stress, dynamic pressure changes, contact with other cell types (endothelial cells, residing spleen and liver macrophages) and a varying $\mathrm{pH}$, $\mathrm{pO}_{2}$ and $\mathrm{pCO}_{2}$. We hypothesized that shear stress may be a driver for maturation and demonstrate here that it is possible to simulate the shear stress component of in vivo circulation using a simple ex vivo circulation mechanism, leading to loss of cell surface area and selective loss of protein content and of mitochondrial content in cultured reticulocytes. Finally, we delineate a novel role for non-muscle myosin IIA (NMIIA) in shear-responsive reticulocyte vesicle transport and maturation. We demonstrate its specific phosphorylation and localization in the proximity of autophagic vesicle markers in reticulocytes and show that chemical inhibition of NMIIA leads to an inability to lose cell volume, as well as a reduction in mitochondrial clearance.

\section{Methods}

\section{Antibodies}

A list of antibodies used in this study is provided in the Online Supplementary Methods.

\section{Native reticulocyte isolation and in vitro erythroid culture}

Native $\mathrm{CD} 71^{+}$reticulocytes and $\mathrm{CD} 71^{-}$erythrocytes were isolated from the red blood cell fraction obtained by Histopaque sepa- ration of healthy donor platelet apheresis waste blood using CD71 MicroBead (Miltenyi Biotec) isolation according to the manufacturer's instructions. In vitro-cultured reticulocytes were differentiated from $\mathrm{CD}_{34}{ }^{+}$cells isolated from the mononuclear cell fraction according to previously published protocols. ${ }^{4}$ All source material was provided with written informed consent for research use given in accordance with the Declaration of Helsinki (NHSBT, Filton, Bristol). The research into the mechanisms of erythropoiesis was reviewed and approved by the Bristol Research Ethics Committee (REC Number 12/SW/0199).

\section{Proteomics experimental design, data acquisition and analysis}

Two experiments were performed: (i) a comparison of erythrocytes, endogenous reticulocytes and in vitro-derived reticulocytes with three biological repeats per cell type, for a total of nine individual samples which were processed through both quantitative tandem mass tag (TMT) proteomics and qualitative $\mathrm{TiO}_{2}$-enriched phosphoproteomics; and (ii) a comparison of circulated and noncirculated in vitro-derived reticulocytes with three biological repeats per condition, for a total of six individual samples, which were processed through quantitative TMT proteomics. Cells were washed three times with phosphate-buffered saline containing 1 $\mathrm{mg} / \mathrm{mL}$ bovine serum albumin and $2 \mathrm{mg} / \mathrm{mL}$ glucose; $2 \times 10^{6}$ cells were counted and used per sample for quantitative TMT proteomics, and $10 \times 10^{6}$ cells for qualitative phosphoproteomics. The mass spectrometry proteomics data have been deposited in the ProteomeXchange Consortium via the PRIDE partner repository with the dataset identifiers PXD009015, PXD009023 and PXD009024. Extended details on the methods for sample preparation, data acquisition and analysis are provided in the Online Supplementary Methods.

\section{Immunofluorescence and automated image processing}

Details on the methods for fixed and live-cell immunofluorescence are provided in the Online Supplementary Methods. For quantitative analysis, tile scans composed of 10x10 images taken at 1024x1024 resolution of cells labeled with Calcein Blue and Mitotracker Deep Red were generated using confocal imaging and analyzed with arivis Vision4D (arivis, Germany). Further details on the analysis are provided in the Online Supplementary Methods.

\section{Sample preparation for rheoscopy and cell analysis}

Two million cells were diluted in $200 \mu \mathrm{L}$ of a polyvinylpyrrolidone solution (polyvinylpyrrolidone viscosity 28.1; Mechatronics Instruments). Samples were assessed in an Automated Rheoscope and Cell Analyzer (ARCA) ${ }^{19}$ consisting of a plate-plate optical shearing stage (model CSS450) mounted on a Linkam imaging station assembly and temperature controlled using Linksys 32 software (Linkam Scientific Instruments). The microscope was equipped with an LMPlanF1 50x with a $10.6 \mathrm{~mm}$ working distance objective (Olympus) illuminated by an X-1500 stroboscope (Vision Light Tech) through a band-pass interference filter (CWL 420 nm, FWHM 10 nm; Edmund Optics). Images were acquired using a uEye camera (UI-2140SE-M-GL; IDS GmbH). At least 1500 valid cells per sample were analyzed using bespoke ARCA analysis software.

\section{Ex vivo cell circulation}

Details on the construction of the circulation system are provided in the Online Supplementary Methods. Cells were packed and resuspended in a mixture of culture medium and Sanquin reticulocyte stabilizer solution (Sanquin Blood Supply, the Netherlands) in a 4:1 ratio. The suspension was then circulated overnight at $37^{\circ} \mathrm{C}$ in $5 \% \mathrm{CO}_{2}$, with the $5 \mathrm{rpm}$ setting. A matched control sam- 
A

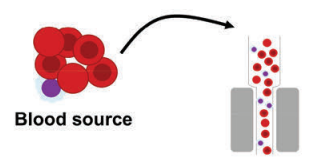

Magnetic separation (CD71+ or CD34+ beads)

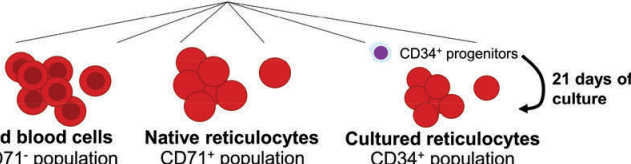

In-depth quantitative analysis using Tandem Mass Tag labeling and nano LC-MS/MS

D

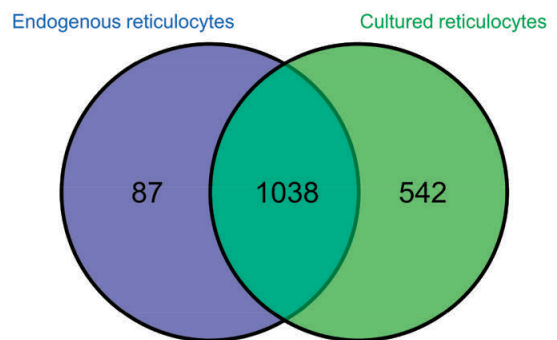

Significantly different proteins compared to Red Blood Cells

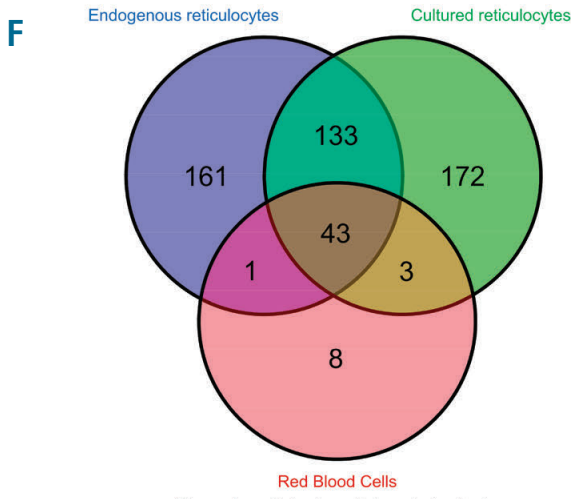

Phosphorylated proteins detected

Figure 1. Reticulocyte culture and maturation lead to differences in phosphorylation status and protein abundance. (A) Experimental design for proteomic comparison - red blood cells, native reticulocytes $\left(\mathrm{CD} 71^{+}\right.$cells) and hematopoietic precursors $\left(\mathrm{CD} 34^{+}\right.$ cells) were separated with magnetic bead isolation. Cultured reticulocytes were leukofiltered after 21 days of culture post-CD $34^{+}$cell isolation. (B) Heatmap visualization of the tandem mass tag (TMT) proteome dataset generated from $\log _{2}$ fold-change values of expression in native and cultured reticulocytes with erythrocytes as a baseline. Individual samples are visualized as separate rows. Blue denotes lower expression, and red denotes higher expression. Proteins were clustered through the average link method and a corresponding dendrogram was generated, shown to the left of the heatmap ( $\mathrm{n}$. of proteins analyzed: 2140 ; false discovery rate $=5 \%$ ). (C) Heatmap visualization of TMT proteome dataset generated from $\log _{2}$ fold-change values of expression in cultured reticulocytes with native reticulocytes as a baseline. The processing was the same as that for Figure 1B. (D) Venn diagram of differentially expressed proteins (absolute log fold-change $>1$ ) in endogenous and cultured reticulocytes using red blood cells as a baseline for comparison. A total of 1667 proteins were differentially expressed in reticulocytes. (E) Heatmap visualization of the "Actin Cytoskeleton and Remodeling" Gene Ontology category present in the TMT proteome dataset, comparing cultured reticulocytes with native reticulocytes as the baseline. The processing was the same as that for Figure 1B. (F) Venn diagram of the phosphorylated proteins detected through qualitative phosphoproteomics comparison of red blood cells, native reticulocytes (NatRet) and cultured reticulocytes (CultRet). The dataset was filtered at a 5\% false discovery rate for this analysis, with no further constraints. A total of 521 phosphoproteins were detected. (G) High-confidence peptides (false discovery rate $=1 \%$ ) detected through qualitative phosphoproteomics. The table was filtered to include only peptides which were present in at least two samples in the same condition. The ID column denotes the UniProt protein identities. Common protein names and protein symbols (GN) are defined in the Protein column. The phosphorylation site of the respective peptides is in the column Site and was acquired through comparison with the PhosphoSitePlus ${ }^{\circledast}$ database. ${ }^{53}$ The number of occurrences per condition of each phosphorylation is written in the rightmost columns.
B
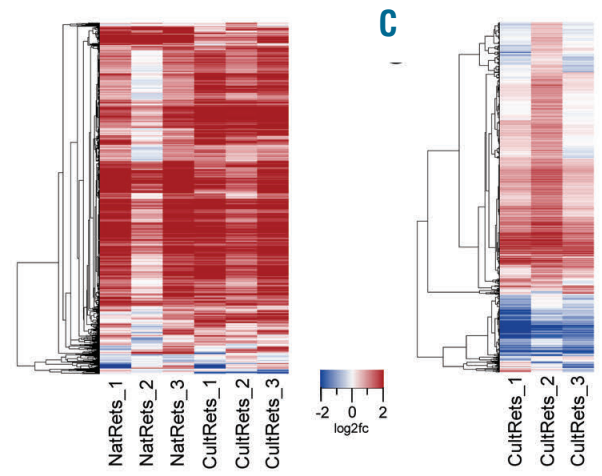

E

G
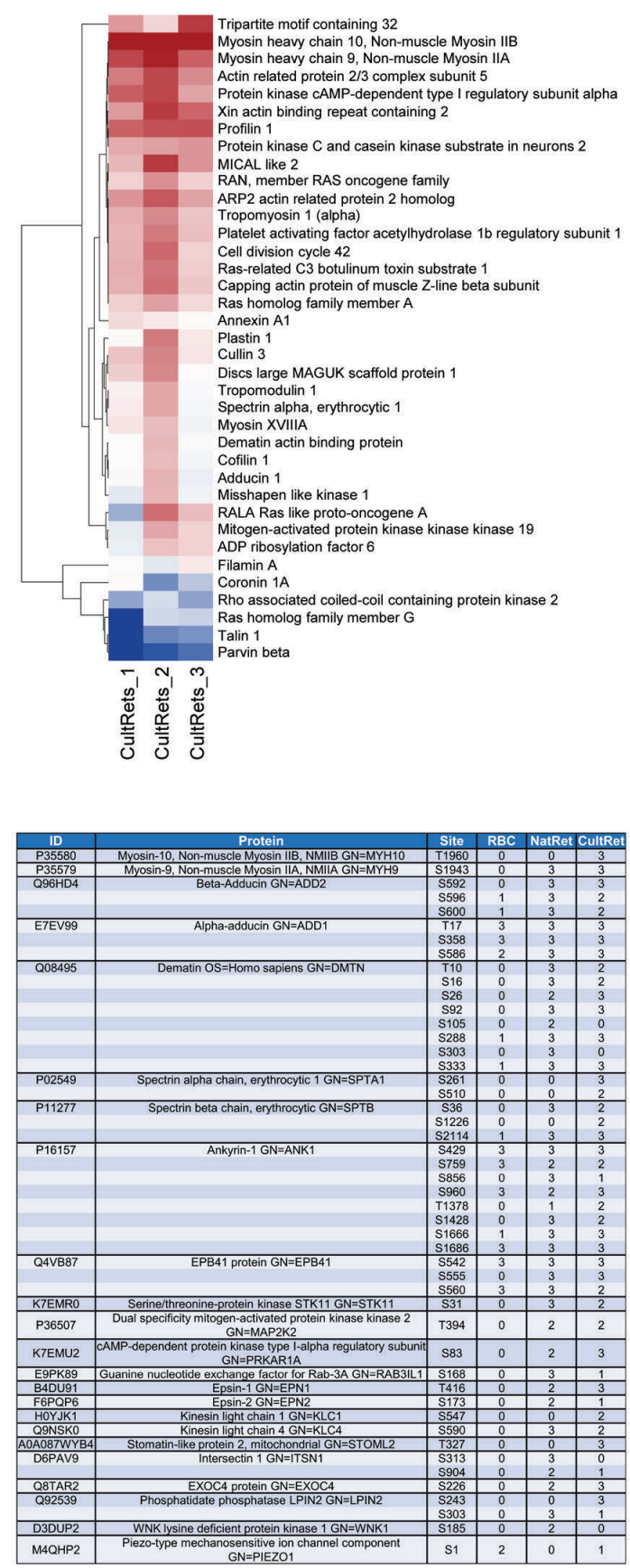
ple was kept in culture without circulation. For drug treatment to test NMIIA inhibition, cells were exposed during circulation to either $20 \mu \mathrm{M}$ blebbistatin(-) (Insight Biotechnology), or $20 \mu \mathrm{M}$ blebbistatin(+) (Source BioScience) as a negative control.

\section{Results}

\section{Quantitative proteomics data analysis reveals} differences between erythrocytes, native reticulocytes and cultured reticulocytes

In order to explore possible changes in protein abundance and function underlying reticulocyte maturation, we produced a TMT-based quantitative proteomics dataset and a qualitative phosphoproteomics dataset comparing donor-matched native reticulocytes, cultured reticulocytes and erythrocytes. The $\mathrm{CD} 71^{+}$population (native reticulocytes) was isolated through magnetic bead isolation. While this strategy does not distinguish between subsets of reticulocytes as compared to alternative fluorescence activated cell sorting-based methods, ${ }^{6}$ it does provide a convenient method to obtain the required number of cells from each separate donor for biochemical analysis. The CD71 population (erythrocytes) was collected from the flow-through of the CD71+ cell isolation, and CD34+ precursors were isolated, expanded and differentiated to generate in vitro-derived (cultured) reticulocytes. The design of this experiment is summarized in Figure 1A. All samples submitted to proteomics were characterized regarding established reticulocyte markers (Online
Supplementary Figure S1). Data resulting from the experiment are presented in Online Supplementary Table S1.

These proteomic datasets were analyzed with the objective of determining possible targets for further study of reticulocyte maturation. First, a broad comparison of protein expression changes between reticulocytes and erythrocytes was performed. In accordance with previous literature, where a loss of protein content is expected in maturation, ${ }^{20,21}$ both native and cultured reticulocytes have a higher abundance of most proteins, as shown in Figure $1 \mathrm{~B}$. However, protein clusters where expression is higher in the erythrocyte can also be observed and were found to encompass both contaminating material (serum proteins and keratins) and functional proteins (e.g. carbonic anhydrase 1/2/3). Only six proteins were significantly increased (average $\log _{2}$ fold-change $>1$ ) in the erythrocyte population, and all corresponded to contamination except for carbonic anhydrase 1 . This observation reflects the fact that residual protein synthesis still takes place in the reticulocyte by means of the ribosomes and RNA present in the cell, and carbonic anhydrase 1 in particular is known to be synthesized during maturation. ${ }^{22}$ Some inter-sample variation was observed between the same cell types, likely due to variable purity in the CD71 isolation protocol (Online Supplementary Figure S1). Furthermore, the heterogeneous nature of reticulocyte populations undergoing maturation creates inherent difficulties for comparative proteomics. Nonetheless, native and in vitro-derived (cultured) reticulocytes were found to exhibit a very similar global protein expression profile (Figure 1C). A summary
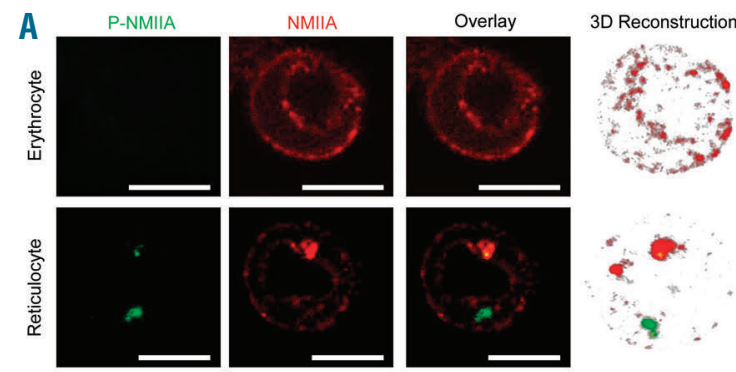

C
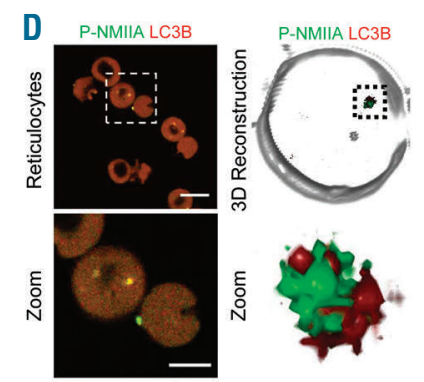

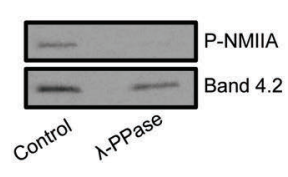

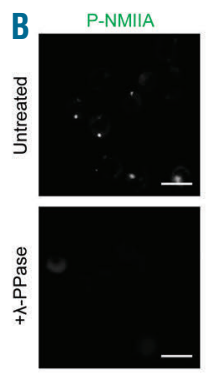
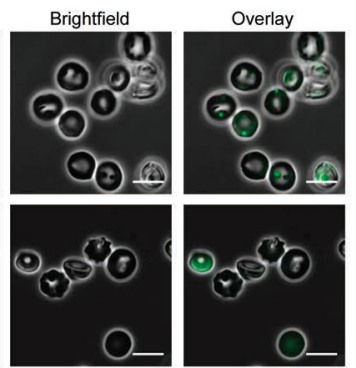

E

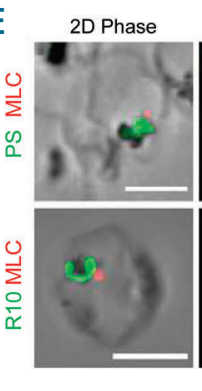

$2 \mathrm{D}$

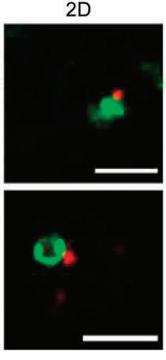

3D Reconstruction

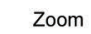

Figure 2. Non-muscle myosin IIA components localize proximally to autophagic vesicles. (A) Erythrocytes (top) and cultured reticulocytes (bottom) were co-labeled for phospho-non-muscle myosin IIA (S1943) (green) and non-muscle myosin IIA (NMIIA) (red) and subjected to confocal imaging. Cells were fixed, labeled and cytospun as described in the Methods section. Scale bars: 10 um. (B) Untreated reticulocytes (top) and reticulocytes treated with lambda phosphatase (bottom) for a dephosphorylation assay were labeled for phospho-NMIIA (S1943) (green). Cells were fixed, treated with lambda phosphatase, labeled and cytospun as described in the Methods section. Scale bars: $10 \mu \mathrm{m}$. (C) Western blot of the phospho-NMIIA signal before and after the dephosphorylation assay with lambda phosphatase. Protein 4.2 was used as a loading control. (D) Cultured reticulocytes were co-labeled with phospho-NMIIA (S1943) (green) and LC3B (red), and imaged by confocal microscopy. A zoomed-in section is shown to facilitate identification of the structures. Three-dimensional reconstruction was done using arivis Vision4D software, maximizing contrast for clarity. Scale bar in initial image: $10 \mu \mathrm{m}$. Scale bar in zoomed-in image: $5 \mu \mathrm{m}$. (E) Fixed and permeabilized cultured reticulocytes were duallabeled for phosphorylated myosin light chain (red) and phosphatidylserine or trypsin sensitive glycophorin A epitope R10 as indicated (green) and imaged using confocal microscopy. For R10 staining, reticulocytes were previously treated with trypsin. Images are shown in two-dimensional phase overlay, two-dimensional fluorescence, three-dimensional reconstructions and three-dimensional reconstructions with zoom highlighted. Scale bars: $5 \mu \mathrm{m}$. 


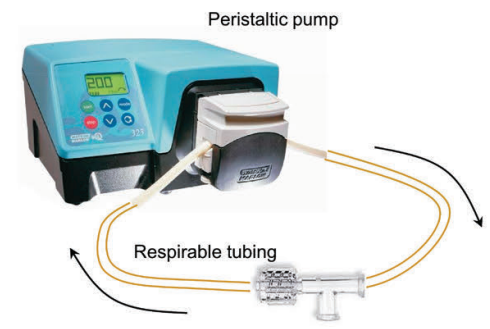

Overnight circulation at $37^{\circ} \mathrm{C}, 5 \% \mathrm{CO}_{2}$
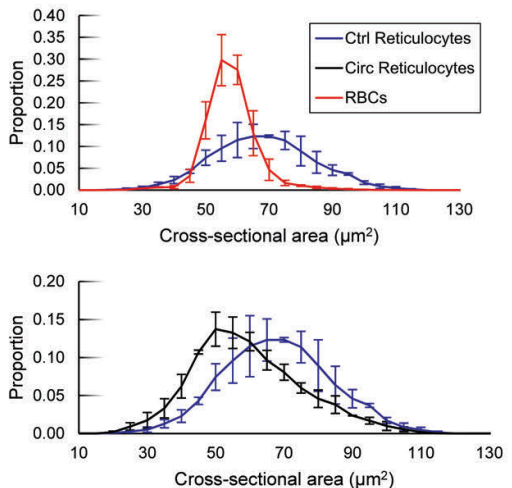

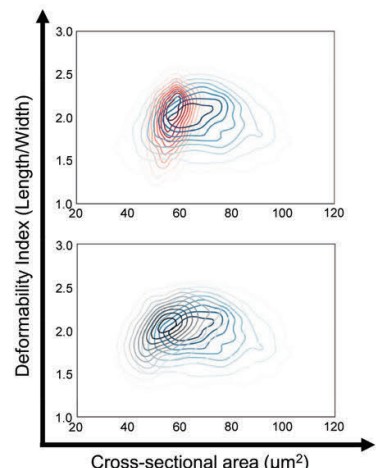

D

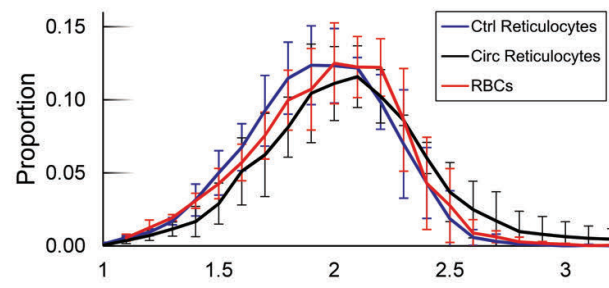

E

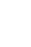

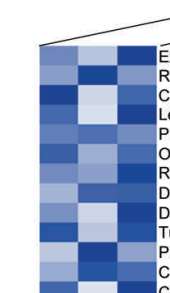
Exocyst complex component $1<$
Ring-box 1

Cytochrome b5 reductase 1

Leucine rich repeat containing 47

Proteasome subunit beta 6

Opioid growth factor recept
Ribosomal protein $\mathrm{S7}$

DNAJ heat shock protein family ( $\mathrm{Hsp} 40)$ member $\mathrm{C5}$

Dimethylarginine
Tubulin alpha 4a

Protein phosphatase, Mg2+/Mn2+ dependent $1 G$

Calcineurin
Calpain 7

Transcription factor A, mitochondrial $\leftarrow$

X-linked Kx blood group

Dishevelled associated activator of morphogenesis 1 Mitochondrial genome maintenance exonuclease 1 Mitochondrial
Calumenin

Ubiquitin protein ligase E3 component n-recognin 7 (putative) PTTG1 interacting protein

Proteasome subunit beta 2

Methionyl aminopeptidase 1

GABA type A receptor
Ribosomal protein $\mathrm{S} 13$

Ribosomal protein $L 23$ a

RAB5A, member RAS oncogene family

Ribosomal protein L29

Nidogen 1

Aquaporin 3 (Gill blood group)

Acidic residue methyltransferase 1

LPS responsive beige-like anchor protein

Ribosomal protein 528

Translocase of outer mitochondrial membrane 20

Profilin 3

RAN binding protein

Protein kinase $C$ theta

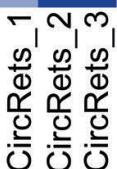

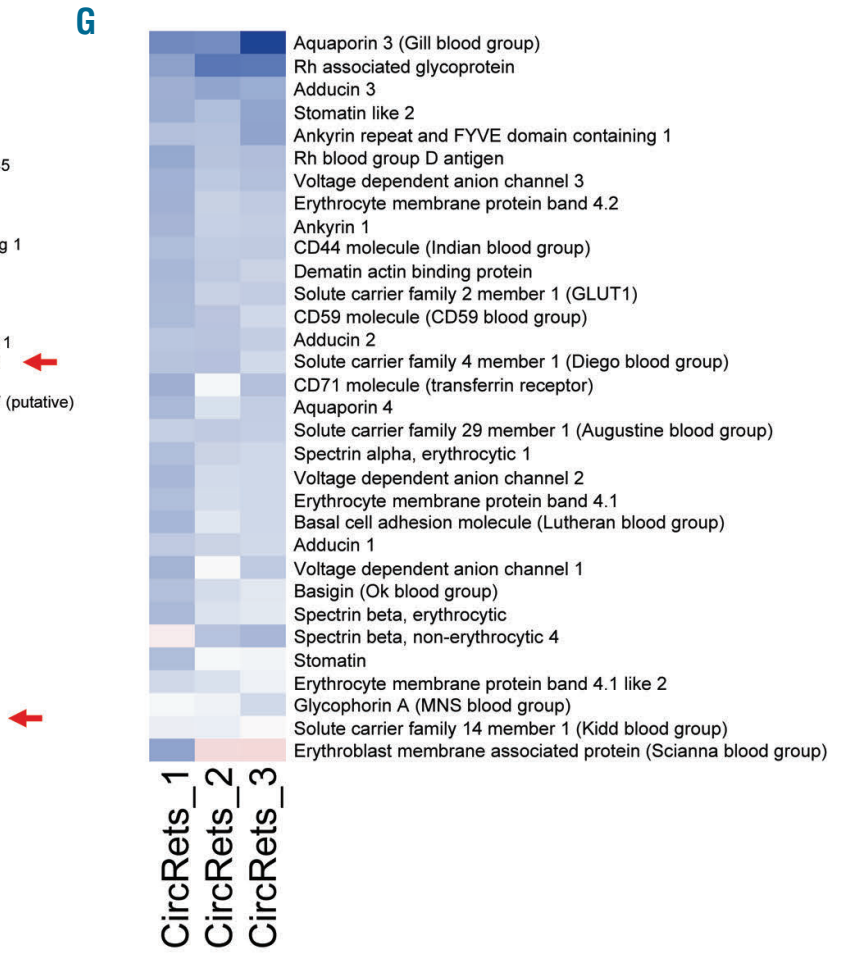

Figure 3. In vitro circulation of cultured reticulocytes recapitulates aspects of reticulocyte maturation. (A) Diagram of the circulation system used to study the impact of shear stress on reticulocyte maturation. Cells are subjected to a continuous difference in pressure with the use of a peristaltic pump, and are circulated overnight at $37^{\circ} \mathrm{C}$ in $5 \% \mathrm{CO}_{2}$. A matched control is kept in culture overnight, without circulation. (B) Cross-sectional area profile of red blood cells, reticulocytes kept in culture overnight without circulation (Ctrl), and reticulocytes circulated overnight (Circ). The profile is plotted as the average of the proportion of cells within each area "bin" (corresponding to $5 \mu \mathrm{m}^{2}$ ). Error bars correspond to the standard deviation of each average. Data were obtained using an Automated Rheoscope and Cell Analyzer (ARCA). (C) ARCA contour plots demonstrating the probability distribution of erythrocytes, uncirculated reticulocytes and circulated reticulocytes with cross-sectional area plotted against deformability index. The probability density functions for the data were generated through kernel-density estimation of three pooled samples per group. (D) Deformability index (length/width ratio) profile of red blood cells, uncirculated reticulocytes and circulated reticulocytes. The profile is plotted as the average of the proportion of cells within each deformability index "bin" (corresponding to 0.1 ratio units). Error bars correspond to the standard deviation of each average.

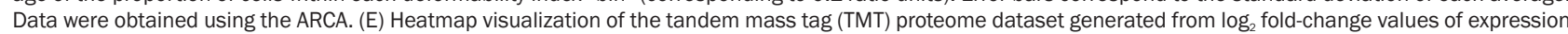
in circulated reticulocytes with uncirculated reticulocytes as a baseline. Individual samples were visualized as separate rows. Blue denotes lower expression, and red denotes higher expression. Proteins are displayed horizontally by order of average $\log _{2}$ fold-change ( $\mathrm{n}$. of proteins analyzed: 2447). (F) Subset of Figure 3D detailing the 40 proteins with the lowest $\log _{2}$ fold-change, or, alternatively, the 40 proteins in which abundance is reduced to the greatest degree in circulated reticulocytes. Mitochondrial proteins are indicated with red arrows. (G) Subset of Figure 3D detailing erythroid proteins of interest. Proteins are displayed vertically by order of average $\log _{2}$ fold-change. 
A

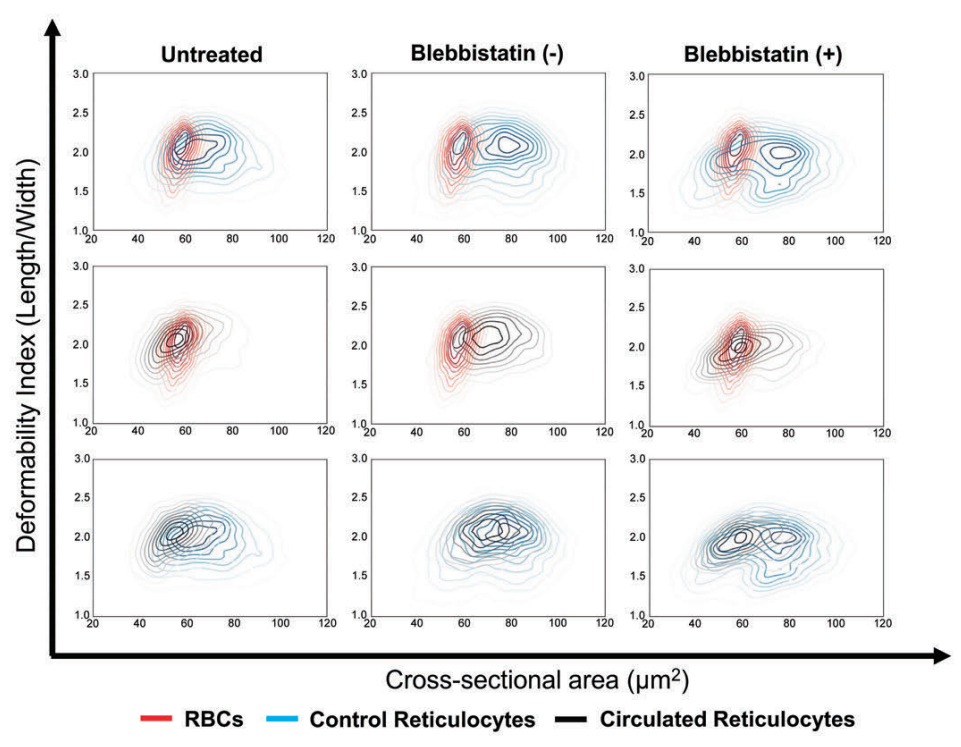

B

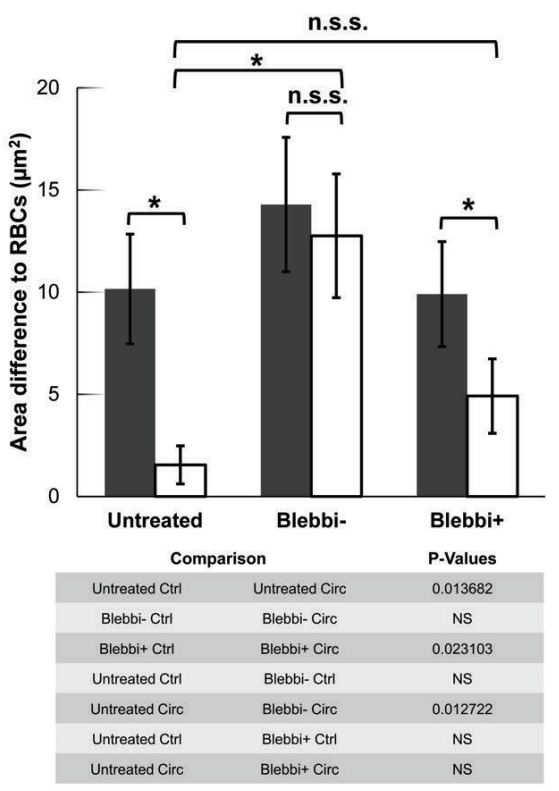

Figure 4. Blebbistatin treatment abrogates circulation-induced differences in reticulocyte cross-sectional area. (A) Automated Rheoscope and Cell Analyzer (ARCA) contour plots demonstrating the probability distribution of erythrocytes, uncirculated reticulocytes and circulated reticulocytes with cross-sectional area plotted against deformability index. Reticulocytes were untreated, treated with blebbistatin(-) or treated with blebbistatin(+) as displayed in each column. Cell types are plotted pairwise for ease of visualization. The probability density functions for the data were generated through kernel-density estimation of three pooled samples per group. (B) Comparison of the area difference to red blood cells between untreated, blebbistatin(-)-treated and blebbistatin(+)-treated cells that were left in culture (gray) or were circulated overnight (white). *Indicates a $P$-value of under 0.05 , with $n$.s.s. (not statistically significant) indicating $P$-values above 0.05 . $P$-values for relevant comparisons are shown underneath the graph. All comparisons were made with a paired two-tailed $t$-test between arrays of area difference to red blood cells. Data are represented as mean \pm standard deviation $(n=3)$.

of significant differences in abundance is shown in Figure 1D. The proteins with the greatest difference in abundance between cultured and native reticulocytes were processed using the STRING database to create proteinprotein interaction networks (Online Supplementary Figure S2), which ease data visualization. It was observed that protein families containing a large number of proteins skewed the resulting networks. Thus, ribosomal proteins, initiation factors and tRNA synthetases were removed from the dataset and the network was re-analyzed using the Ingenuity Pathway Analysis software. The top scoring networks are summarized in Online Supplementary Table S2 and the top four networks are shown in Online Supplementary Figure S3.

One of the most striking features observed in cultured reticulocytes compared to native reticulocytes was a global increased abundance of metabolic enzymes. Particularly, enzymes related to glycolysis and processing of ornithine were significantly upregulated (Online Supplementary Figure S4), in accordance with previous reports. ${ }^{23,24}$ We hypothesize that the nutrient abundance present in culture media may cause the observed metabolic changes, rather than an inherent difference between native and cultured reticulocytes.

Interestingly, the Ingenuity Pathway Analysis software also showed underlying differences in the abundance of various cytoskeletal proteins between native and cultured reticulocytes. Since the process of reticulocyte maturation requires extensive remodeling of the cytoskeleton, ${ }^{5}$ the Gene Ontology category of 'Actin Cytoskeleton and Remodeling' was further scrutinized (Figure 1E). We confirmed significant differences in the abundance of several proteins within this category, most notably non-muscle myosin IIA and IIB (NMIIA/MYH9 and NMIIB/MYH10) and talin-1. Loss of both myosin ${ }^{7}$ and talin- $1^{21}$ has been previously associated with reticulocyte maturation, which leads us to hypothesize that cultured reticulocytes may represent an intermediate maturational stage compared to native reticulocytes, with additional stimuli required to complete the maturation process.

Post-translational modification of proteins via phosphorylation provides an additional level of regulation beyond that achieved through differences in protein expression and is of particular importance within the transcriptionally deficient red blood cell. Phosphorylation of membrane and cytoskeletal proteins is already known to influence red blood cell membrane properties and protein interactions ${ }^{7,25-27}$ and increased phosphorylation of the cytoskeletally-associated junctional protein complex component protein $4.1 \mathrm{R}$ has previously been reported in murine reticulocytes compared to erythrocytes. ${ }^{7}$ In order to assess the protein phosphorylation landscape of human reticulocytes compared to erythrocytes, qualitative phosphoproteomics data for the same samples were acquired and are summarized in the diagram shown in Figure 1F, with the full dataset in Online Supplementary Table S3. The table in Figure $1 \mathrm{G}$ summarizes high-confidence data (false discovery rate $=1 \%$, proteins with one single peptide across all samples disregarded), in which it can be observed that many proteins are phosphorylated exclusively in reticulocytes, consistent with their higher kinase activity. ${ }^{28}$

\section{Non-muscle myosin IIA localizes proximally to vesicle compartments in the reticulocyte}

As the most highly upregulated cytoskeletal proteins in the cultured compared to native reticulocytes, myosins 
were selected for further investigation. Coincidentally, both NMIIA and IIB were consistently phosphorylated in native and/or cultured reticulocyte samples, but not in erythrocytes. Interestingly, the occurrence of phosphorylation at the S1943 site on NMIIA has previously been implicated in cargo binding in natural killer cells. ${ }^{29}$ Since reticulocyte maturation involves vesicle extrusion, ${ }^{4}$ we examined whether phospho-NMIIA could be the driving force behind this activity.

Using a well-characterized commercially available phospho-residue specific antibody we co-labeled fixed and permeabilized reticulocytes for phospho-NMIIA together with its non-phosphorylated form. Non-phosphorylated NMIIA localize at the plasma membrane (Figure 2A, Online Supplementary Figure S5), as anticipated based upon its known integration with the spectrin-actin cytoskeleton. ${ }^{30}$ However, phosphorylated NMIIA localized to punctae that were observed to be exclusive to reticulocytes. The distinct localization of phospho-NMIIA in reticulocytes was particularly striking, and we therefore decided to validate the signal of the phospho-antibody through a dephosphorylation assay with lambda phosphatase. After lambda phosphatase treatment, the phospho-NMIIA signal was largely abrogated in the reticulocyte (Figure 2B), and this result was confirmed by western blotting (Figure 2C). Unexpectedly, not all phosphoNMIIA-positive structures were found to co-label with non-phosphorylated NMIIA. We speculate that this may reflect differing binding accessibility of the antibodies used against the phosphorylated and non-phosphorylated forms of the protein.

To further investigate the localization of phosphoNMIIA and to identify the adjacent vesicular compartment, reticulocytes were co-labeled with an antibody specific to the lipidated form of LC3B, an established marker of autophagic membranes. ${ }^{31}$ Figure $2 \mathrm{D}$ shows fluorescent labeling of phospho-NMIIA immediately proximal to the autophagic vesicles. Phosphorylated myosin light chain (S20, MLC), a marker for increased myosin activity, ${ }^{32}$ was then detected with additional vesicle markers as shown in Figure 2E. Using protease treatment methodology to detect membrane proteins that have been internalized from the plasma membrane, ${ }^{33}$ phosphorylated MLC was found adjacent to glycophorin A-positive vesicles inside the cell, emerging from the cell and in trypsin-treated reticulocytes. These combined data suggest that NMIIA may have a role in the movement of vesicles in the reticulocyte.

\section{In vitro circulation of cultured reticulocytes simulates the maturation process}

The most striking difference between in vitro cell culturing systems and the conditions to which maturing reticulocytes are exposed in the body is the absence of shear stress caused by circulation in the bloodstream. Therefore, to investigate the effects of this influencing factor, we attempted to construct a system that could induce shear stress conditions similar to those of in vivo circulation. Microcirculation systems have previously been used to study the effects of circulation in other cell types, ${ }^{34,35}$ however, our objective was to build a relatively inexpensive and scalable system. Thus, rather than use lithography for the creation of microcapillary-scale tubing (as this would limit the volumes used), we decided to use wider and more flexible gas-permeable tubing. The final system has a very simple design and can be constructed with readily available parts, as shown in the diagram (Figure $3 \mathrm{~A}$ ).

Deformability and cross-sectional area of unstimulated reticulocytes and erythrocytes were measured using an Automated Rheoscope and Cell Analyzer (ARCA) ${ }^{19}$ and the measurements were used as a basis to assess alterations in these parameters in response to circulation. Figure $3 \mathrm{~B}$ demonstrates a decrease in cross-sectional area to a range more akin to that observed for erythrocytes following overnight circulation of reticulocytes, providing important evidence that circulatory shear stress ex vivo is able to induce alterations in the morphology of reticulocytes associated with maturation. Figure $3 \mathrm{C}$ shows that the erythrocyte and reticulocyte populations are visually distinct when the cross-sectional area and deformability measurements are combined, with the distribution of circulated reticulocytes approaching the distribution of erythrocytes. Figure 3D shows no reduction in the deformability index profile, confirming that the cells maintain their functional viability and do not form microspherocytes.

Matched control and circulated reticulocytes were submitted for TMT-based quantitative proteomics (Online Supplementary Table S4), which showed that the global outlook is of reduced protein content after circulation (Figure 3E). However, not all proteins decreased in the same manner. As expected, the amount of CD71 decreased following circulation, albeit incompletely and to a variable degree between replicates. Figure $3 \mathrm{~F}$ shows that the most greatly decreased proteins after circulation include a significant number of mitochondrial and ribosomal proteins. Erythroid-specific proteins of the dataset are illustrated in Figure $3 \mathrm{G}$, showing that the decrease is also consistent with previous literature on the protein changes inherent to reticulocyte maturation in mice $^{7}$ (e.g. lower decreases of glycophorin A, band 4.1, 4.2 and spectrins compared to adducin). Of note, a large number of serum-derived proteins were shown to exhibit increased abundance within the samples prepared from circulated compared to uncirculated reticulocytes. Since the cells were incubated in identical media, it is likely that circulation results in increased adhesion of these proteins to the extracellular membrane of the reticulocytes. Although this is likely inconsequential, we cannot exclude a role for serum protein binding in facilitating maturation, therefore, a detailed list of these proteins is provided in Online Supplementary Figure S6.

\section{Non-muscle myosin IIA inhibition significantly affects} the response of reticulocytes to shear stress

Having established a system that can be used to characterize the response of cultured reticulocytes to shear stress, we next tested the impact of blocking NMIIAmediated activity with the use of blebbistatin, a selective and high-affinity inhibitor of NMIIA and NMIIB which preferentially binds to the ATPase intermediate and slows down phosphate release. ${ }^{36}$ Blebbistatin is particularly useful for experiments of this nature because of the existence of both an active and an inactive enantiomer, blebbistatin(-) and blebbistatin(+), respectively, which allows for the use of a matched control. It has been reported that NMIIB levels are undetectable through western blotting of red blood cells, with NMIIA being the predominant isoform in human erythrocytes. ${ }^{37}$ Therefore, although bleb- 
A

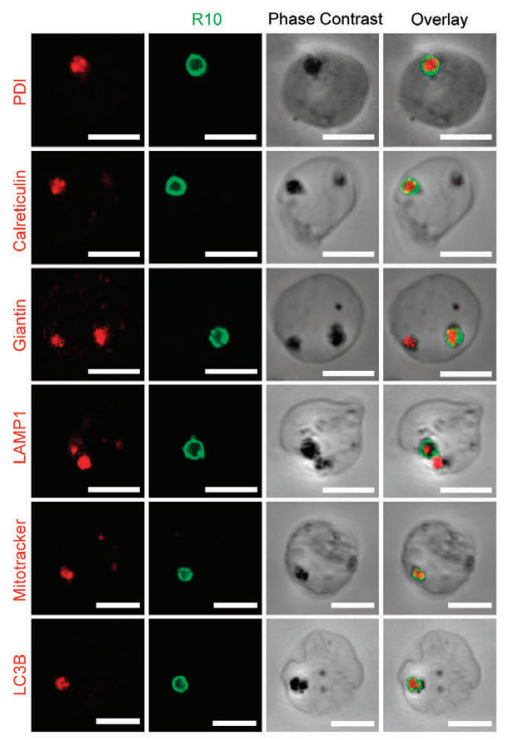

B

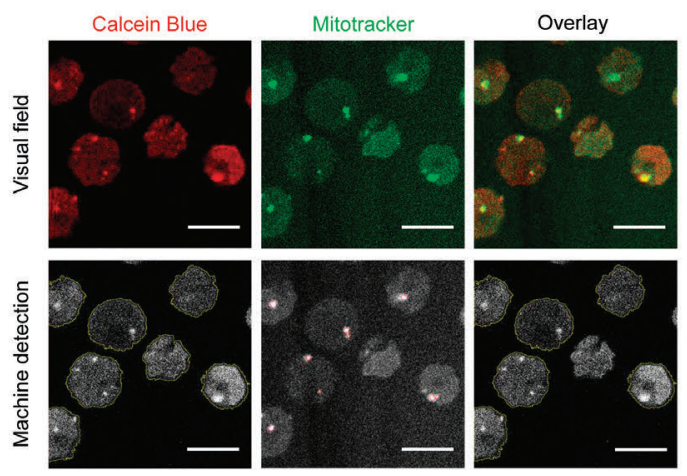

C

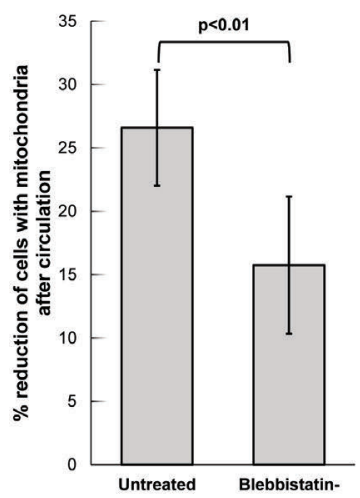

Figure 5. Blebbistatin treatment abrogates circulation-induced differences in reticulocyte vesicle content. (A) Permeabilized trypsin-treated reticulocytes were probed with R10 (pseudo-colored green) and protein disulfide isomerase (PDI), calreticulin, giantin, LAMP-1, Mitotracker or LC3B (pseudo-colored red) and imaged using confocal microscopy. Scale bars: $5 \mu \mathrm{m}$. (B) Example of the machine detection algorithm used for quantitative imaging of cells containing mitochondria (Online Supplementary Methods). Images were obtained using confocal imaging. The top row shows the individual channels corresponding to Calcein Blue (pseudo-colored red) and Mitotracker (pseudo-colored green), and an overlay of the channels. In the bottom row machine detection of the Calcein Blue signal is shown through yellow borders surrounding detected cells, machine detection of the Mitotracker signal is shown with red borders surrounding detected mitochondria, and cells containing mitochondria are shown with yellow borders in the overlay column. Scale bars: $10 \mu \mathrm{m}$. (C) Comparison of the reduction in the proportion of cells with mitochondria after circulation using automated counting, in both untreated reticulocytes and blebbistatin(-)-treated reticulocytes. Data are represented as mean \pm standard deviation $(n=3)$.

bistatin is unable to distinguish between NMII isoforms, its effect is far more likely to have an impact on NMIIA activity.

Thus, we analyzed reticulocyte deformability and circulation-mediated area loss after overnight circulation concurrent with the use of blebbistatin enantiomers. Figure 4A summarizes the multiple experiments performed. Use of either enantiomer did not affect reticulocyte deformability in a significant manner; however, the reduction in cross-sectional area resulting from circulation was diminished to non-significant levels in reticulocytes treated with blebbistatin(-). Interestingly, Smith et al. previously reported an increase in deformability of blebbistatin-treated erythrocytes as assessed through use of a microfluidic device ${ }^{37}$ in contrast to our ARCA-based observations on reticulocytes. This may reflect differences in the specific parameters assessed by each method as well as variances in the intrinsic cytoskeletal properties of the two cell types. Paired comparison graphs of the cross-sectional area and deformability of untreated, blebbistatin(-)-treated and blebbistatin(+)-treated reticulocytes are shown in Online Supplementary Figure S7. Statistical analysis of the data with pairwise comparisons of relevant pairs is shown in Figure 4B.

After having determined that NMIIA inhibition leads to a decrease in the capacity of the reticulocyte to respond to shear stress, the underlying mechanism causing this difference was investigated. As the quantitative proteomics comparison between circulated and uncirculated reticulocytes showed a significant loss of mitochondrial proteins following circulation, we decided to characterize the impact of circulation on the mitochondrial content of the reticulocyte. First, in order to confirm that mitochondrial content in the reticulocyte is associated with vesicular localization (as has been previously described ${ }^{4}$ ), permeabilized, trypsin-treated reticulocytes were co-labeled with $\mathrm{R} 10$ (an antibody to glycophorin A with trypsin-sensitive epitope) and markers for mitochondria (Mitotracker), endoplasmic reticulum proteins (calreticulin and PDI), Golgi (giantin), lysosomes (LAMP-1) and autophagosomes (LC3B). Vesicles with contents positive for all of these markers were observed (Figure $5 \mathrm{~A})$. Cells stained with PDI antibody were manually quantified through the use of Vision4D software before and after circulation for the presence of PDI-positive vesicles, with a significant decrease ( 3 -fold, $P<0.05$ ) in the percentage of vesicle-containing cells being observed after circulation (Online Supplementary Figure S8).

To further explore the possibility of increased vesicle clearance upon circulation in a high-throughput quantitative manner, a high-resolution live-imaging protocol was developed using Vision4D software, which facilitates algorithm-based segmentation in large planes created from individual image stitching. ${ }^{38}$ For this, reticulocytes were co-labeled with Calcein Blue $\mathrm{AM}^{\mathrm{TM}}$ and the live cell imaging-compatible mitochondrial marker Mitotracker Deep Red FM ${ }^{\mathrm{TM}}$, both cell-permeable dyes. Calcein Blue was used to avoid problems related to edge detection in bright-field images, as internal edges are typically displayed in reticulocytes due to their irregular structure. The Calcein Blue-labeled cell was then used as a binary image mask for the detection of internal mitochondria. Figure 5B shows the algorithm's method with a representative immunofluorescence image. Despite the presence of a fluorescent background in both channels used, our method is able to successfully identify cells in the image, mitochondria in the cells, and recognize cells that contain mitochondria. Automated image processing in this way also avoids user-created bias.

Having developed this protocol, circulated reticulocytes 
were left untreated or were treated with blebbistatin(-). In all cases, a reduction in the percentage of cells with mitochondria upon circulation was identified, which was significantly affected by treatment with blebbistatin(-), as shown in Figure 5C. Thus, we have demonstrated a link between mitochondrial loss and NMII activity. In concert with the observation of mitochondrial co-localization with vesicle markers, proximity of NMIIA to those same vesicle markers and the impact of NMIIA activity in the loss of cross-sectional area in the circulating reticulocyte, we have demonstrated a functional link between NMIIA activity and vesicle clearance in reticulocyte maturation.

\section{Discussion}

The study of the process of human reticulocyte maturation has been massively enhanced by the ability to generate large numbers of late reticulocytes by laboratory culture methods. ${ }^{3}$ To date, much of the published literature has focused on comparison of in vitro-derived reticulocytes to circulating mature erythrocytes. ${ }^{20,21,39}$ Here we report the first quantitative protein abundance and qualitative phosphoproteomic datasets comparing human donor-matched adult native reticulocytes, erythrocytes and in vitro culturederived reticulocytes. These data highlight the broad proteomic equivalence of cultured reticulocytes to their natively derived counterparts and provide a basis for more detailed exploration of maturation processes and mechanisms that occur in vivo and in vitro.

In order to explore the contribution of circulatory shear stress to the maturation of reticulocytes and dissect this mechanical element from that of cell-cell interactions and other features of the in vivo circulatory system, a simple circulation system was developed that is easily adaptable and was shown to have a significant impact on reducing cross-sectional area, vesicle/mitochondrial content and general protein content of the circulated in vitro-derived reticulocytes in a way that recapitulates their natural progression through maturation.

These ex vivo data highlight the influence that shear stress is able to exert on biological processes fundamental to reticulocyte maturation. We demonstrate that while the mechanical process of circulation results in a consistent reduction of cell volume and protein abundance, circulated reticulocytes maintain partial expression of the transferrin receptor, CD71. Loss of CD71 has been described in the literature as occurring via exosome release, a process which is independent from autophagosome release. ${ }^{8}$ Thus, our data provide further evidence for these pathways being uncoupled in maturation.

Importantly, these data demonstrate that shear stress is necessary but not sufficient to generate a fully mature erythrocyte and suggest that cell-cell interactions or other influencing aspects present during in vivo circulation are required to facilitate the complete maturation process.

In addition to loss of cell volume, loss of organellar content is one of the distinguishing hallmarks of reticulocyte maturation in vivo, and here also shown to be stimulated by ex vivo circulation. Organelles such as mitochondria are cleared from the reticulocyte in a process partially dependent on autophagy..$^{40}$ The presence of autophagic vacuoles in human reticulocytes was described by Kent et al. back in $1966 .{ }^{41}$ Subsequent studies demonstrated that the maturation of late circulating $\mathrm{R} 2$ reticulocytes involves the generation of endocytic vesicles which fuse with autophagosomes to create large autophagic vesicles, ${ }^{4}$ corresponding to the vacuoles described by Kent et al. ${ }^{41}$ However, the process leading to transport and extrusion of these vesicles is currently undefined.

Proteomic profiling of native and in vitro-derived reticulocytes identified NMIIA as being among the most differentially expressed proteins in these cells compared to mature erythrocytes. NMIIA interacts with actin to contribute to various cellular processes, such as cell migration, ${ }^{42}$ adhesion ${ }^{43}$ and cytokinesis. ${ }^{44}$ NMIIA has also been implicated in autophagosome maturation and lysosome fusion through association with autophagy-related receptors. ${ }^{45}$ In erythroid cells, the NMIIB isoform has been described as having an essential role in enucleation of the differentiating erythroblast. ${ }^{46}$ However, there is currently no defined role for NMIIA in erythroid cells other than as a component of the cytoskeleton. ${ }^{37}$ We show that reticulocytes exhibit a NMIIA phosphorylation that has been previously associated with vesicle transport ${ }^{29}$ and is undetectable in the mature red blood cell, both by mass spectrometric analysis and by immunofluorescence.

Phosphorylation of the S1943 site has been associated with filament destabilization, ${ }^{47}$ which could be necessary for the regulation of NMIIA assembly dynamics. ${ }^{48}$ Phosphorylated NMIIA localizes proximally to LC3B, a known autophagic vesicle marker which was observed to colocalize with other known erythroid autophagic vesicle markers. Moreover, the phosphorylated myosin light chain was found in a similar localization. The observed concurrent localization of the phosphorylated NMIIA heavy chain and the active light chain in proximity to reticulocyte vesicles led us to speculate that NMIIA is responsible for autophagic vesicle movement in the maturing reticulocyte. We explored this hypothesis through pharmacological inhibition of NMII activity by blebbistatin, a selective and potent inhibitor, and show that while inhibition of NMII activity does not affect reticulocyte viability or capacity to deform, it leads to a significant decrease in the cell's ability to respond to shear stress by loss of cell volume and autophagosome-mediated mitochondrial clearance.

Finally, it is notable that shear stress-induced calcium ion $\left(\mathrm{Ca}^{2+}\right)$ influx is a well-described phenomenon in erythrocytes ${ }^{49,50}$ as well as other cell types. ${ }^{51,52}$ NMIIA activity is regulated by phosphorylation of its light chain, which in turn is regulated by $\mathrm{Ca}^{2+}$ influx and interaction with calmodulin. ${ }^{32}$ It is therefore attractive to speculate that shear stress-mediated induction of $\mathrm{Ca}^{2+}$ influx may indirectly modulate NMIIA activity and thereby influence vesicle transport in the reticulocyte. However, further work is required to confirm this hypothesis.

In conclusion, our results have uncovered a previously undescribed mechanism of shear stress response in the human reticulocyte which is dependent on NMII activity for vesicle clearance and cell volume reduction.

\section{Acknowledgments}

We would like to thank Dr. Kate Heesom and Dr. Marieangela Wilson, as well as the proteomics facility of the University of Bristol, for proteomics sample processing and data acquisition. We also thank Dr. Emile van den Akker and Sanquin (Amsterdam, the Netherlands) for providing the Sanquin reticulocyte stabilizing reagent, Matthias Rust (arivis, Rostock, Germany) for the training provided with Vision4D, Dr. 
Jiandi Wan (Rochester Institute of Technology, New York, USA), for helpful discussions on the setup of an ex vivo circulation system and the Watson-Marlow Fluid Technology Group for permission to reproduce an image of their pump systems in Figure $3 A$.

\section{Funding}

This work was funded by the European Union (F.A. H2020MSCA-ITN-2015, "RELEVANCE", Grant agreement N. 675117), NHS Blood and Transplant (NHSBT) R\&D grants
(WP15-04 and WP15-05) and a National Institute for Health Research Blood and Transplant Research Unit (NIHR BTRU) in Red Blood Cell Products at the University of Bristol in partnership with NHSBT (NIHR-BTRU-2015-10032). We acknowledge the Wolfson Bioimaging Facility of the University of Bristol for use of their confocal systems, as well as the MRC for establishing the Facility and the BBSRC Alert 13 capital grant (BB/L014181/1) for funding the acquisition of the Leica SP8. The views expressed are those of the authors and not necessarily of the NHS, the NIHR or the Department of Health.

\section{References}

1. Bessman JD. Reticulocytes. In: Walker HK, Hall WD, Hurst JW, eds. Clinical Methods: The History, Physical, and Laboratory Examinations. Boston, 1990

2. Gifford SC, Derganc J, Shevkoplyas SS, Yoshida T, Bitensky MW. A detailed study of time-dependent changes in human red blood cells: from reticulocyte maturation to erythrocyte senescence. Br J Haematol. 2006;135(3):395-404

3. Giarratana MC, Rouard H, Dumont A, et al. Proof of principle for transfusion of in vitro-generated red blood cells. Blood. 2011;118(19):5071-5079.

4. Griffiths RE, Kupzig S, Cogan N, et al. Maturing reticulocytes internalize plasma membrane in glycophorin A-containing vesicles that fuse with autophagosomes before exocytosis. Blood. 2012;119(26): 6296-6306.

5. Chasis JA, Prenant M, Leung A, Mohandas N. Membrane assembly and remodeling during reticulocyte maturation. Blood. 1989;74(3):1112-1120.

6. Malleret B, Xu F, Mohandas N, et al. Significant biochemical, biophysical and metabolic diversity in circulating human cord blood reticulocytes. PLoS One. 2013;8(10):e76062

7. Liu J, Guo X, Mohandas N, Chasis JA, An $\mathrm{X}$. Membrane remodeling during reticulocyte maturation. Blood. 2010;115(10):20212027.

8. Blanc L, Vidal M. Reticulocyte membrane remodeling: contribution of the exosome pathway. Curr Opin Hematol. 2010;17(3): 177-183.

9. Koury MJ, Koury ST, Kopsombut P, Bondurant MC. In vitro maturation of nascent reticulocytes to erythrocytes. Blood. 2005;105(5):2168-2174.

10. Johnstone RM, Adam M, Hammond JR, Orr L, Turbide C. Vesicle formation during reticulocyte maturation. Association of plasma membrane activities with released vesicles (exosomes). J Biol Chem. 1987;262 (19):9412-9420.

11. Lee E, Choi HS, Hwang JH, Hoh JK, Cho $\mathrm{YH}$, Baek EJ. The RNA in reticulocytes is not just debris: it is necessary for the final stages of erythrocyte formation. Blood Cells Mol Dis. 2014;53(1-2):1-10.

12. Blanc L, Liu J, Vidal M, Chasis JA, An X, Mohandas $\mathrm{N}$. The water channel aquaporin-1 partitions into exosomes during reticulocyte maturation: implication for the regulation of cell volume. Blood. 2009;114(18): 3928-3934.
13. Stolz A, Ernst A, Dikic I. Cargo recognition and trafficking in selective autophagy. Nat Cell Biol. 2014;16(6):495-501

14. Holm TM, Braun A, Trigatti BL, et al. Failure of red blood cell maturation in mice with defects in the high-density lipoprotein receptor SR-BI. Blood. 2002;99(5):18171824.

15. Zhang J, Ney PA. Role of BNIP3 and NIX in cell death, autophagy, and mitophagy. Cell Death Differ. 2009;16(7):939-946.

16. King JS, Veltman DM, Insall RH. The induction of autophagy by mechanical stress. Autophagy. 2011;7(12):1490-1499.

17. Holroyde CP, Gardner FH. Acquisition of autophagic vacuoles by human erythrocytes. Physiological role of the spleen. Blood. 1970;36(5):566-575.

18. Kupzig S, Parsons SF, Curnow E, Anstee DJ, Blair A. Superior survival of ex vivo cultured human reticulocytes following transfusion into mice. Haematologica. 2017;102(3):476-483.

19. Dobbe JG, Streekstra GJ, Hardeman MR, Ince $C$, Grimbergen CA. Measurement of the distribution of red blood cell deformability using an automated rheoscope. Cytometry. 2002;50(6):313-325.

20. Prenni JE, Vidal M, Olver CS. Preliminary characterization of the murine membrane reticulocyte proteome. Blood Cells Mol Dis. 2012;49(2):74-82.

21. Chu TTT, Sinha A, Malleret B, et al. Quantitative mass spectrometry of human reticulocytes reveal proteome-wide modifications during maturation. Br J Haematol. 2018;180(1):118-133.

22. Meyers NL, Brewer GJ, Tashian RE. Enzymatic synthesis of carbonic anhydrases by human reticulocytes. Biochim Biophys Acta. 1969;195(1):176-185.

23. Srivastava A, Evans KJ, Sexton AE, Schofield L, Creek DJ. Metabolomics-based elucidation of active metabolic pathways in erythrocytes and HSC-derived reticulocytes. J Proteome Res. 2017;16(4):14921505.

24. Darghouth D, Giarratana MC, Oliveira I et al. Bio-engineered and native red blood cells from cord blood exhibit the same metabolomic profile. Haematologica. 2016;101(6):e220-222.

25. Manno S, Takakuwa Y, Mohandas N. Modulation of erythrocyte membrane mechanical function by protein 4.1 phosphorylation. J Biol Chem. 2005;280(9): 7581-7587.

26. Gauthier E, Guo X, Mohandas N, An X. Phosphorylation-dependent perturbations of the 4.1R-associated multiprotein complex of the erythrocyte membrane.
Biochemistry. 2011;50(21):4561-4567

27. Koshino I, Mohandas N, Takakuwa Y Identification of a novel role for dematin in regulating red cell membrane function by modulating spectrin-actin interaction. J Bio Chem. 2012;287(42):35244-35250.

28. Fairbanks G, Palek J, Dino JE, Liu PA Protein kinases and membrane protein phosphorylation in normal and abnormal human erythrocytes: variation related to mean cell age. Blood. 1983;61(5):850-857.

29. Sanborn KB, Mace EM, Rak GD, et al. Phosphorylation of the myosin IIA tailpiece regulates single myosin IIA molecule association with lytic granules to promote NK-cell cytotoxicity. Blood. 2011;118(22): 5862-5871.

30. Basu A, Harper S, Pesciotta EN, Speicher KD, Chakrabarti A, Speicher DW. Proteome analysis of the triton-insoluble erythrocyte membrane skeleton. J Proteomics. 2015;128:298-305.

31. Hansen TE, Johansen T. Following autophagy step by step. BMC Biol. 2011;9:39.

32. Mizuno Y, Isotani E, Huang J, Ding H, Stull JT, Kamm KE. Myosin light chain kinase activation and calcium sensitization in smooth muscle in vivo. Am J Physiol Cell Physiol. 2008;295(2):C358-364.

33. Mankelow TJ, Griffiths RE, Trompeter S, et al. Autophagic vesicles on mature human reticulocytes explain phosphatidylserinepositive red cells in sickle cell disease. Blood. 2015;126(15):1831-1834.

34. Wan J, Fan R, Emery $T$, et al. In vitro microfluidic circulatory system for circulating cancer cells. Protoc Exch. 2016;2016.

35. Chen Y, Chan HN, Michael SA, et al. A microfluidic circulatory system integrated with capillary-assisted pressure sensors. Lab Chip. 2017;17(4):653-662.

36. Kovacs M, Toth J, Hetenyi C, MalnasiCsizmadia A, Sellers JR. Mechanism of blebbistatin inhibition of myosin II. J Biol Chem. 2004;279(34):35557-35563.

37. Smith AS, Nowak RB, Zhou S, et al Myosin IIA interacts with the spectrinactin membrane skeleton to control red blood cell membrane curvature and deformability. Proc Natl Acad Sci USA. 2018;115(19):E4377-E4385

38. Gutzeit E, Scheel C, Dolereit T, Rust M Contour based split and merge segmentation and pre-classification of zooplankton in very large images. Proceedings of the 2014 9th International Conference on Computer Vision Theory and Applications (Visapp), Vol 1. 2014;417-424.

39. Wilson MC, Trakarnsanga K, Heesom KJ, et al. Comparison of the proteome of adult 
and cord erythroid cells, and changes in the proteome following reticulocyte maturation. Mol Cell Proteomics. 2016;15(6):19381946.

40. Zhang J, Ney PA. Reticulocyte mitophagy: monitoring mitochondrial clearance in a mammalian model. Autophagy. 2010;6(3):405-408.

41. Kent G, Minick OT, Volini FI, Orfei E. Autophagic vacuoles in human red cells. Am J Pathol. 1966;48(5):831-857.

42. Vicente-Manzanares M, Ma X, Adelstein RS, Horwitz AR. Non-muscle myosin II takes centre stage in cell adhesion and migration. Nat Rev Mol Cell Biol. 2009;10(11):778-790.

43. Ebrahim S, Fujita T, Millis BA, et al. NMII forms a contractile transcellular sarcomeric network to regulate apical cell junctions and tissue geometry. Curr Biol. 2013;23 (8):731-736.

44. Newell-Litwa KA, Horwitz R, Lamers ML.
Non-muscle myosin II in disease: mechanisms and therapeutic opportunities. Dis Model Mech. 2015;8(12):1495-1515.

45. Tang HW, Wang YB, Wang SL, Wu MH, Lin SY, Chen GC. Atg1-mediated myosin II activation regulates autophagosome formation during starvation-induced autophagy. EMBO J. 2011;30(4):636-651

46. Ubukawa K, Guo YM, Takahashi M, et al. Enucleation of human erythroblasts involves non-muscle myosin IIB. Blood. 2012;119(4):1036-1044

47. Dulyaninova NG, Malashkevich VN, Almo SC, Bresnick AR. Regulation of myosin-IIA assembly and Mts1 binding by heavy chain phosphorylation. Biochemistry. 2005;44 (18):6867-6876.

48. Betapudi V, Gokulrangan G, Chance MR, Egelhoff TT. A proteomic study of myosin II motor proteins during tumor cell migration. J Mol Biol. 2011;407(5):673-686.

49. Brain MC, Pihl C, Robertson L, Brown CB.
Evidence for a mechanosensitive calcium influx into red cells. Blood Cells Mol Dis. 2004;32(3):349-352

50. Danielczok JG, Terriac E, Hertz L, et al. Red blood cell passage of small capillaries is associated with transient $\mathrm{Ca}(2+)$-mediated adaptations. Front Physiol. 2017;8:979.

51. Ando J, Komatsuda T, Kamiya A Cytoplasmic calcium response to fluid shear stress in cultured vascular endothelial cells. In Vitro Cell Dev Biol. 1988;24(9):871-877.

52. Ikeda $Y$, Handa $M$, Kamata $T$, et al Transmembrane calcium influx associated with von Willebrand factor binding to GP $\mathrm{Ib}$ in the initiation of shear-induced platelet aggregation. Thromb Haemost. 1993;69(5): 496-502.

53. Hornbeck PV, Zhang B, Murray B, Kornhauser JM, Latham V, Skrzypek E. PhosphoSitePlus, 2014: mutations, PTMs and recalibrations. Nucleic Acids Res. 2015;43(Database issue):D512-520. 